\title{
Type-III intermittency in a four-level coherently pumped laser
}

\author{
J. Pujol and M. Arjona \\ Departament d'Optica i Optometría, Universitat Politecnica de Catalunya, E-08222 Terrassa, Spain \\ R. Corbalán \\ Departament de Física, Universitat Autoǹoma de Barcelona, E-08193 Bellaterra, Spain
}

(Received 16 December 1992)

\begin{abstract}
We study a homogeneously broadened four-level model for a coherently pumped laser with pump and laser fields having crossed linear polarizations. For a parameter range of the type explored in the experiments by Tang et al. [Phys. Rev. A 44, R35 (1991)] the system exhibits a family of type-III-intermittency transitions to chaos in which the onset of intermittency is preceded by period-2, period-3, or period-4 states. We find similarities but also differences between the results of our theory and their experimental results.

PACS number(s): 42.60.Mi, 42.50.Lc, 42.55. - f
\end{abstract}

\section{INTRODUCTION}

The occurrence of nonlinear dynamics and chaotic behavior are well-established phenomena that affect many systems belonging to different fields such as physics, chemistry, or biology. Moreover, systems seemingly very different may exhibit some kind of universal behavior. Thus, it is well known that there are at least three universal routes from regular to chaotic motion: The Ruelle-Takens-Newhouse scenario, the Feigenbaum scenario or period-doubling route, and the PomeauManneville scenario or intermittency route. The intermittency route is characterized by chaotic bursts ("turbulent" phases) that appear at random times, interrupting the otherwise nearly regular oscillations ("laminar" phases). Three types of intermittency are known, which depend on the way the destabilization of the limit cycle associated with the regular oscillations takes place [1].

Lasers are among the most interesting physical systems for the study of the dynamic behavior of nonlinear dissipative systems and their transitions to chaos [2]. Particularly relevant in this respect are coherently pumped lasers, which have allowed the first experimental observation [3] of a behavior remarkably similar to the predictions of the paradigmatic Lorenz-Haken model [4], including period-doubling transitions to chaos, and also the first observation in an optical system of a family of transitions to chaos by type-III intermittency [5] and, more recently, by type-I intermittency also [6]. These observations were all performed on the same coherently pumped $\mathbf{N H}_{3}$ far-infrared laser, operating in different domains of the control-parameter space. Sacher, Elsäser, and Göbel reported recently the first experimental observation of type-II intermittency in an optical system using a $\mathrm{GaAs} / \mathrm{Al}_{x} \mathrm{Ga}_{1-x} \mathrm{As}$ semiconductor laser with external feedback [7]. We focus in this paper in the modeling of laser dynamics of intermittency type III.

In almost all the dynamical models the laser field is considered as a scalar quantity coupled to a medium transition between two nondegenerated levels whose population inverts an incoherent pumping mechanism. These models are therefore unable to describe two important physical aspects of the ammonia laser used in the experiments $[3,5,6]$. On the one hand, the $81-\mu \mathrm{m}$ laser transition in $\mathrm{NH}_{3}$ is optically pumped with the $P(13)$ line of the $\mathrm{N}_{3} \mathrm{O}$ laser via an adjacent transition sharing the upper level with the lasing transition. It is therefore coherently excited and associated effects such as Autler-Townes splitting or Raman two-photon processes may result in new dynamic features [8]. On the other hand, the ammonia laser operated with pump and generated fields with crossed linear polarizations [5]. This is also an important aspect since it is known [9] that $\mathrm{NH}_{3}$ laser transitions with linear polarizations parallel to that of the pump laser exhibit dramatically different dynamics. To incorporate the vectorial character of the light field into the laser model one has to consider also the level degeneracy related to the angular momentum of the molecular levels.

We developed recently a model that takes into account both the coherent optical pumping and the influence of light polarization on the laser dynamics [10]. This model has proven its capacity to reproduce the Lorenz-like dynamics of the $\mathrm{NH}_{3}$-laser experiments [11]. Here we use this model in an attempt to reproduce the type-III intermittency observed on the same $\mathrm{NH}_{3}$ laser [5]. In our numerical results we find qualitatively some of the main signatures of the dynamics observed by Tang, Pujol, and Weiss, but a complete understanding of the experimental behavior is not achieved.

\section{MODEL}

Figure 1 illustrates the $J_{a}=0 \rightarrow J_{b}=1 \rightarrow J_{c}=0$ transition scheme assumed for the homogeneously broadened gain medium of an optically pumped ring laser. It is the simplest level scheme that enables one to consider interaction with pump $\mathbf{E}_{2}(z, t)$ and generated $\mathbf{E}_{1}(z, t)$ fields composed by arbitrary superpositions of right and left circularly polarized components. Both fields are considered as uniform plane monochromatic waves propagating along the optical $(z)$ axis of the ring resonator 


$$
\mathbf{E}_{j}(z, t)=\sum_{\mu= \pm} \mathrm{e}^{\mu} A_{j}^{\mu}(t) \exp \left\{i\left[k_{j} z-v_{j}^{\mu} t-\phi_{j}^{\mu}(t)\right]\right\} / 2+\text { c.c. } \quad(j=1,2)
$$

where the unit vectors $\mathrm{e}^{ \pm}=\mp\left(\mathbf{e}_{x} \pm i \mathbf{e}_{y}\right) / \sqrt{2}$. The $z$ axis is also chosen to be the quantization axis, so that only four levels are involved [the sublevel $\left(J_{b}=1, m=0\right)$ does not couple to the fields], which we shall simply denote as $a$,,+- , and $c$. In the following we restrict to the case of $M$-degenerate levels ( $\epsilon=0$, see Fig. 1) and fully resonant pump field $\left(v_{2}^{+}=v_{2}^{-}=\Omega_{2}\right)$. The amplitudes $A_{2}^{\mu}$ and phases $\phi_{2}^{\mu}$ of the pump beam are considered as constant in time control parameters. For the reference frequencies of the generated field we choose $v_{1}^{+}=v_{1}^{-}=\omega_{c}$, being the closest empty cavity resonance frequency. The unknown amplitudes $A_{1}^{\mu}$ and phases $\phi_{1}^{\mu}$ of the generated beam are slowly varying functions of time. We assume that the pump beam drives the $a-b$ transition only, and the generated field the $b-c$ transition only. The field-matter coupling is characterized by four real Rabi frequencies (see Fig. 1) defined as $\beta^{\mu}=d_{2} A_{2}^{\mu} / 2 \hbar$ and $\alpha^{\mu}=d_{1} A_{1}^{\mu} / 2 \hbar$ with $d_{j}(j=1,2)$ being molecular transition electric-dipole moments. As in the experiment [5] we will restrict ourselves here to studying the case of pump and laser fields with crossed linear polarizations. For this case one has $\phi_{j}^{+}=\phi_{j}^{-}=\phi_{j}(j=1,2), \alpha^{+}=\alpha^{-}=\alpha$, and $\beta^{+}=-\beta^{-}=\beta$. In the usual rotating-wave approximation, using the semiclassical density-matrix formalism [12], the Maxwell-Schrödinger equations for our laser system are [11]

$$
\begin{aligned}
& \dot{\rho}_{a a}=\gamma_{a}\left(\rho_{a a}^{0}-\rho_{a a}\right)+4 \beta y_{b a}, \\
& \dot{\rho}_{b b}=\gamma_{\|}\left(\rho_{b b}^{0}-\rho_{b b}\right)+2 \alpha y_{c b}-2 \beta y_{b a}, \\
& \dot{N}_{b c}=\gamma_{\|}\left[\left(\rho_{b b}^{0}-\rho_{c c}^{0}\right)-N_{b c}\right]+6 \alpha y_{c b}-2 \beta y_{b a}, \\
& \dot{y}_{b a}=-\gamma_{a b} y_{b a}+\beta\left(N_{b a}-x_{+-}\right), \\
& \dot{\rho}_{c b}=-\left[\gamma_{\perp}+i\left(\Delta_{1}^{c}+\dot{\phi}\right)\right] \rho_{c b}-i \alpha\left(N_{b c}+x_{+-}\right), \\
& \dot{x}_{+}=-\Gamma x_{+-}+2 \beta y_{b a}+2 \alpha y_{c b}, \\
& \dot{\alpha}=-\chi \alpha-g y_{c b}, \\
& \dot{\phi}=g x_{c b} / \alpha,
\end{aligned}
$$

where $\rho_{j j}$ represents the population of level $j$, $\rho_{b b} \equiv \rho_{++} \equiv \rho_{--}, N_{i j} \equiv\left(\rho_{i i}-\rho_{j j}\right)$ is a population inversion, and $\rho_{i j}$ is the slowly varying complex amplitude of the coherence associated with the transition $i \rightarrow j$ $(i, j=a,+,-, c), x_{i j}=\operatorname{Re}\left(\rho_{i j}\right)$, and $y_{i j}=\operatorname{Im}\left(\rho_{i j}\right)$. Dissipative processes are described by means of population relaxation rates $\gamma_{i}$, coherence relaxation rates $\gamma_{i j}$, and cavity

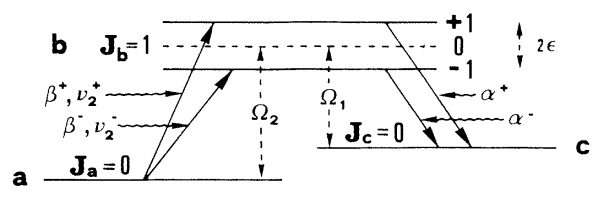

FIG. 1. Schematic representation of the level system and laser fields considered in this work. $2 \beta^{ \pm}$represent the pump Rabi frequencies and $2 \alpha^{ \pm}$the generated ones. loses $\gamma^{1}(l=+,-)$. Here we choose the relaxation rates appropriate for the experimental conditions of Tang, $\mathrm{Pu}$ jol, and Weiss [5], referring to a $\mathrm{NH}_{3}$ pressure $p$ : $\gamma_{c+}=\gamma_{c-} \equiv \gamma_{\perp} ; \quad \gamma_{\perp} / p=6.8 \times 10^{5} \quad \mathrm{sec}^{-1} / \mathrm{Pa} ; \quad \gamma_{+}=\gamma_{-}$ $=\gamma_{c} \equiv \gamma_{\|}=0.28 \gamma_{\perp} ; \quad \gamma_{a}=\gamma_{a c}=0,95 \gamma_{\perp} ; \gamma_{a+}=\gamma_{a-} \equiv \gamma_{a b}$ $=0.95 \gamma_{\perp}$. For $\gamma_{+-} \equiv \Gamma$ no experimental estimation of its value exists; somewhat arbitrarily we choose in the following $\Gamma \equiv 0.5 \gamma_{\perp}$. We assumed equal losses for both components of the generated field $\gamma^{+}=\gamma^{-} \equiv \chi$. For the population of levels in the absence of fields we assumed $[10,11] \quad \rho_{a a}^{0}: \rho_{++}^{0}: \rho_{--}^{0}: \rho_{c c}^{0}=0.9594: 0.0113: 0.0113: 0.0180$. $\Delta_{1}^{c} \equiv\left(\omega_{c}-\Omega_{1}\right)$ is the cavity detuning, and $g \equiv \Omega_{1} d_{1}^{2} N_{0} / 2 \epsilon_{0} \hbar$ represents the unsaturated gain parameter, $N_{0}$ being the number of active molecules per unit volume.

\section{NUMERICAL RESULTS AND DISCUSSION}

We have performed a numerical integration of Eqs. (2) using a seventh- to eighth-order Runge-Kutta method. As mentioned in the Introduction, it is known [10,11] that our equations reveal the existence of periodic and chaotic regimes, with transitions to chaos occurring, for increasing pump field strength $\beta$, and for increasing cavity detuning $\Delta_{1}^{c}$, through an inverse sequence of perioddoubling bifurcations as in the Lorenz-Haken model [4]. A more detailed scanning of the parameter space has allowed us to find also type-III intermittencies close to periodic windows in the chaotic domain. It is interesting to note that in the Lorenz-Haken laser model intermittencies appear also close to periodic windows, but they are of type I $[2,4]$ while in our model they are of type III.

Figure 2 shows the sequence of time dependences of the laser output that we obtain as the pump field intensity is increased from Fig. 2(a) to Fig. 2(e), for a ${ }^{14} \mathrm{NH}_{3}$ gas pressure of $8 \mathrm{~Pa}$ and a fixed resonator tuning. At $\beta / \gamma_{\perp}=0.17$ the system is in a period-3 window [Fig. 2 (c)]. By varying $\beta$ from the center of the window in one direction or in the other one obtains chaos through, respectively, a period-doubling sequence [Figs. 2(b) and $2(\mathrm{a})]$ or an intermittency road [Figs. 2(d) and 2(e)]. The time evolution shown in Fig. 2(d) has the characteristics of type-III intermittency. An inspection of this figure shows that during the laminar phases the intensity of one component of the period-3 pulses decreases while the intensity of the other two components grow. This behavior is time inverted in comparison with the one experimentally observed [5], where the largest peak of the period-3 pulses grows and the two smaller ones decrease. The laminar phases have a random time duration and are separated by chaotic bursts of fairly regular duration. As the pump strength $\beta$ is further increased the average duration of the laminar phases decreases and finally the system reaches the chaotic state shown in Fig. 2(e).

The same behavior is also obtained with fixed pump intensity varying the resonator detuning as shown in Fig. 3, which corresponds to a gas pressure $p=6 \mathrm{~Pa}$ and a pump 

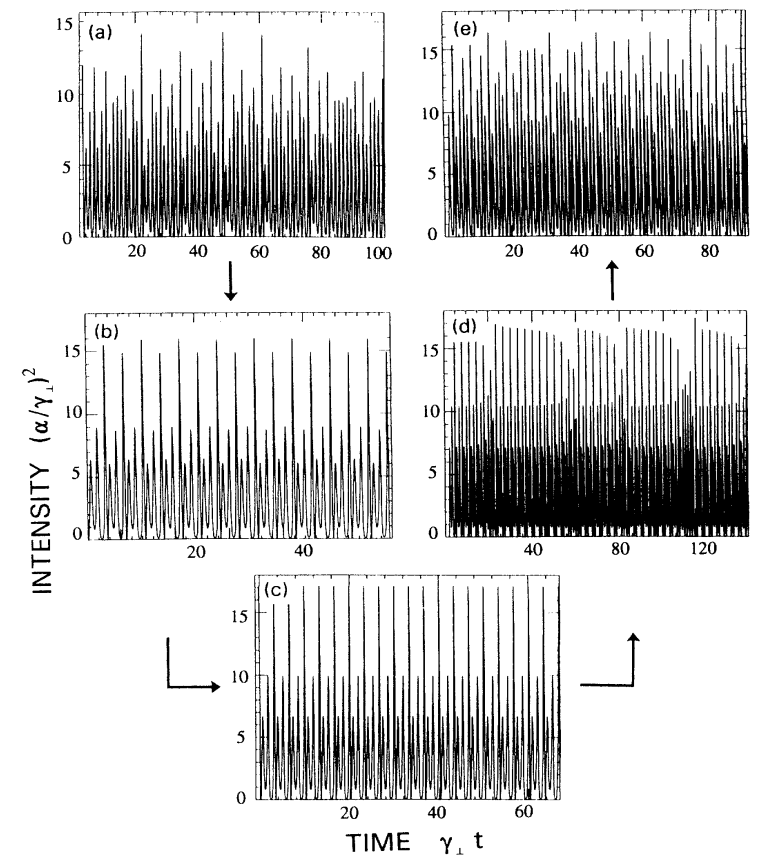

FIG. 2. Dynamic behavior of the laser emission for increasing values of the pump field strength $\beta$. (a) $\beta=0.15 \gamma_{1}$, (b) $\beta=0.16 \gamma_{\perp}$, (c) $\beta=0.17 \gamma_{\perp}$, (d) $\beta=0.173 \gamma_{\perp}$, (e) $\beta=0.18 \gamma_{\perp}$. The gas pressure is $8 \mathrm{~Pa}, g=9214 \gamma_{\perp}^{2}, \Delta_{1}^{c}=0.5 \gamma_{\perp}$, and $\chi=5.2 \gamma_{1}$. Other parameters are given in the text.

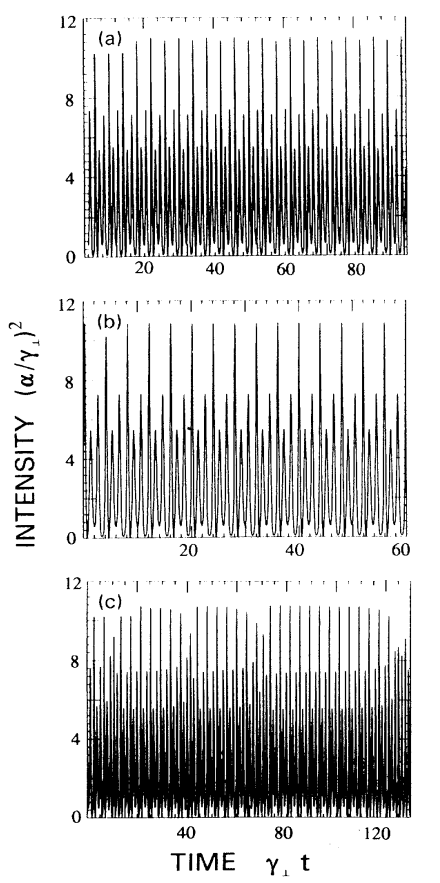

FIG. 3. Time dependence of the laser emission as the resonator detuning $\Delta_{1}^{c}$ is changed. (a) $\Delta_{1}^{c}=0.95 \gamma_{1}$, (b) $\Delta_{1}^{c}=0.99 \gamma_{1}$, (c) $\Delta_{1}^{c}=1.02 \gamma_{\perp}$. The gas pressure is $6 \mathrm{~Pa}, g=12000 \gamma_{1}^{2}, \beta=0.12 \gamma_{\perp}$, and $\chi=5 \gamma_{\perp}$. (Other parameters are as in Fig. 2.) The chaotic states preceding the period- 6 state (a) and following the intermittency pulsing state (c) are not shown for brevity. field $\beta=0.12 \gamma_{\perp}$. The cavity detuning increases from Fig. 3(a) to Fig. 3(c). The chaotic states preceding the period6 state [Fig. 3(a)] and following the intermittency pulsing state [Fig. 3(c)] are not shown for brevity.

As in the experiment we have also found intermittencies preceded by period- 2 and period- 4 states, but not the ones preceded by a period-5 state which were also observed [5]. For a gas pressure of $10 \mathrm{~Pa}$ and a cavity tuned to resonance, increasing the pump strength $\beta$ the system exhibits an inverse period-doubling route to chaos [Fig. $4(a)]$, with period-4 and period- 2 windows in the chaotic domain. By slightly increasing $\beta$ from the center of the period-4 window [Fig. 4(b)] one observes a transition to a chaotic state (not reported) preceded by the intermittency pulsing shown in Fig. 4(c). In this intermittent regime the two larger peaks of the period-4 pulses increase during the laminar phases while the two smaller peaks decrease. This is the type of behavior observed experimentally, however, while in the numerical results the larger peaks of the period- 4 pulses are the first and the third; they are the first and the second in the experiment. By slightly decreasing $\beta$ from the center of the period- 4 win-
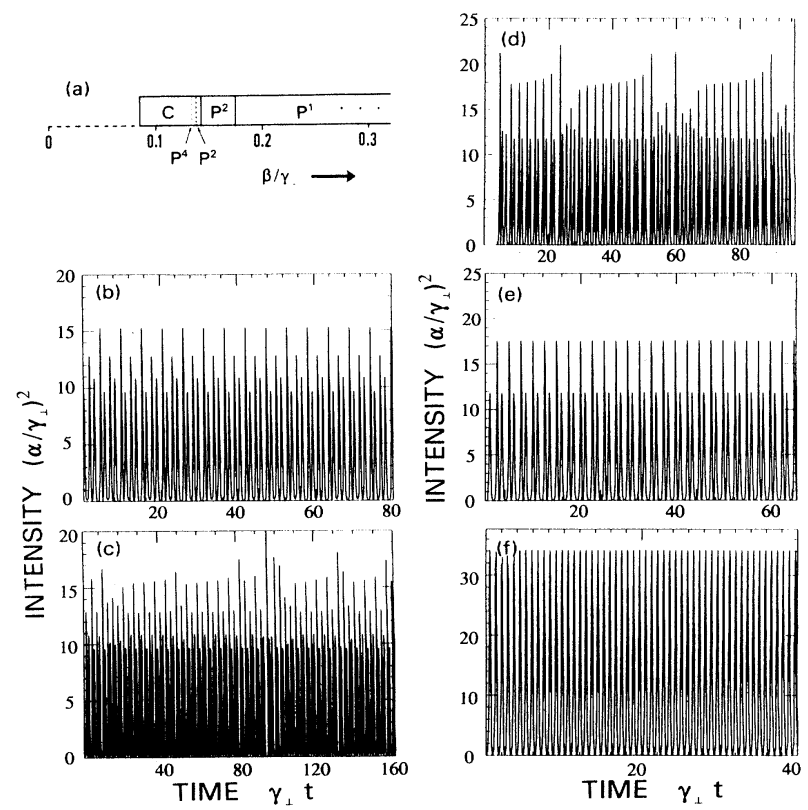

FIG. 4. Dynamic behavior of the laser output for an $\mathrm{NH}_{3}$ gas pressure of $10 \mathrm{~Pa}, g=7285 \gamma_{1}^{2}$, and $\chi=2.85 \gamma_{\perp}$. (a) Bifurcation diagram of the resonantly tuned laser intensity $\alpha^{2}(t)$ for increasing pump field strength $\beta$. $C$ represents chaotic emission, and $P^{n}$ period- $n$ pulsing. Note the appearance of period- 4 and period-2 windows within the chaotic domain. (b) Time dependence of the laser intensity in the period-4 window at $\beta=0.135 \gamma_{\perp}$. (c) Time dependence of type-III intermittency preceded by the period- 4 state for $\beta=0.13505 \gamma_{\perp}$. The chaotic states preceding and following the states reported in (b) and (c), respectively, are not shown for brevity. (d) Time dependence of intermittency at $\beta=0.1485 \gamma_{\perp}$ and $\Delta_{1}^{c}=0$, close to the period-2 window indicated in (a). Keeping $\beta$ fixed and increasing $\Delta_{1}^{c}$ the system first reaches a period-2 state (e) for $\Delta_{1}^{c}=0.2 \gamma_{\perp}$ and then a period-1 state (f) for $\Delta_{1}^{c}=3 \gamma_{\perp}$. 
dow one again obtains a period-doubling route to chaos (not reported in Fig. 4). Similar results can be obtained with different sets of parameters. Figure 5 shows the sequence of time variations of the laser output for a gas pressure of $4 \mathrm{~Pa}$ and a fixed pump intensity, using the resonator tuning as control parameter. Note that the pulsing in this period-4 window [Fig. 5(b)] is different from the one previously discussed.

Close to the period-2 window indicated in Fig. 4(a), decreasing or increasing $\beta$ we again obtain transitions to chaos either through a period-doubling sequence or through the type-III-intermittency pulsing shown in Fig. 4(d), respectively. Starting from this intermittency pulsing and increasing the cavity detuning $\Delta_{1}^{c}$ from zero the system follows the sequence shown in Figs. 4(e) and 4(f). At low cavity detuning we observe a regular period-2 pulsing [Fig. 4(e)]. A further increase of $\Delta_{1}^{c}$ leads to a new simplification of the dynamics of the laser, which now pulses periodically [Fig. 4(f)]. A sequence of this type was also found in the experiment, but with the intermittent state preceded by a chaotic state [5]. In the numerical results we cannot find the chaotic state because the dynamic behavior of the resonantly pumped laser depends only on $\left|\Delta_{1}^{c}\right|$ and not on the sign of the detuning, therefore we cannot further reduce $\Delta_{1}^{c}$ from zero, the value at which appears at the intermittency, in search of a more complex behavior.

In order to reinforce the evidence that the behavior predicted by our model represents type-III intermittency, we have analyzed the statistical distribution $P(t)$ of the
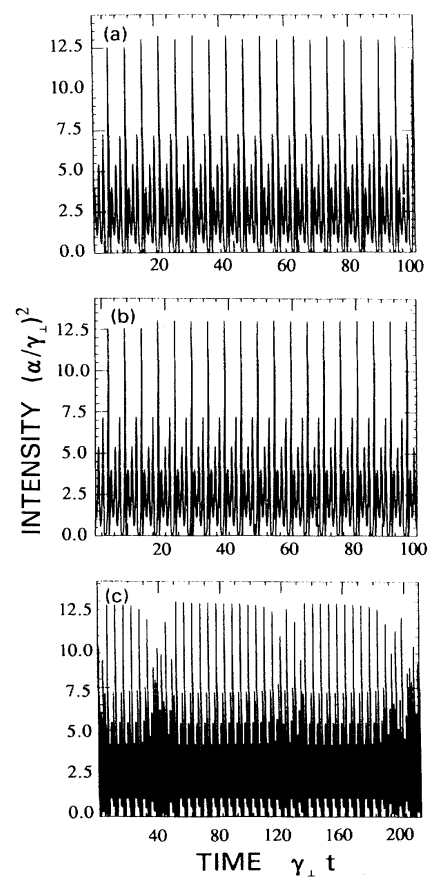

FIG. 5. Time dependence of the laser output as the resonantor tuning is varied. Gas pressure is $4 \mathrm{~Pa}, g=18000 \gamma_{1}^{2}$, $\beta=0.1 \gamma_{1}$, and $\chi=5.2 \gamma_{1}$. (a) Period-8 state. $\Delta_{1}^{c}=0.37 \gamma_{\perp}$. (b) Period-4 state. $\Delta_{1}^{c}=0.4 \gamma_{1}$. (c) Intermittency preceded by a period-4 state. $\Delta_{1}^{c}=0.5 \gamma_{\perp}$. duration of the laminar phases corresponding to the intermittent states shown in Figs. 2(d), 4(d), and 5(c). In making this statistical analysis we have used data sets containing 950 consecutive laminar periods. Figures 2(d), 4(d), and 5(c) are samples of these large data sets. According to [13], for type-III intermittency the fraction $N$ of laminar phases lasting longer than $t_{0}$ is given by

$$
\begin{aligned}
N\left(t>t_{0}\right) & =\int_{t_{0}}^{\infty} d t P(t) \\
& \propto\left\{\epsilon /\left[\exp \left(4 \epsilon t_{0}\right)-1\right]\right\}^{1 / 2},
\end{aligned}
$$

where $\epsilon$ is the bifurcation parameter. The dependence of $N$ on $t_{0}$ is completely characterized by the bifurcation parameter $\epsilon$.

Figure 6 shows that the distribution of the laminarphase durations predicted by our model exhibits the expected feature of the type-III intermittency: the points, calculated from the large data sets mentioned above, agree very well with the line obtained from Eq. (3) for $\epsilon=0.060 \pm 3 \%$ [Fig. 6(a)], $\epsilon=0.027 \pm 4 \%$ [Fig. 6(b)], and $\epsilon=0.019 \pm 3.5 \%$ [Fig. 6(c)], that correspond to the intermittent states preceded by period-3, period-2, and period-4 states shown in Figs. 2(d), 4(d), and 5(c), respectively. Notice that in Eq. (3) $\epsilon$ and $t_{0}$ are dimensionless quantities. Therefore the value of $\epsilon$ obtained from the fitting of Eq. (3) to the numerical points strongly depends on the units used to normalize the time. As in the rest of the paper, for Fig. 6 we have measured it in units of $\left(\gamma_{\perp}\right)^{-1}$. If, as in the experiment [5], we measure the time
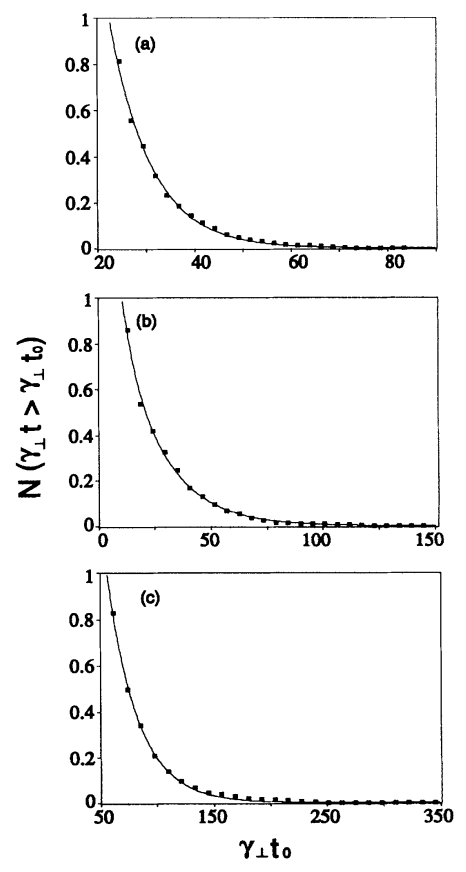

FIG. 6. Distribution of the fraction of laminar phases lasting longer than $t_{0}$. The points are calculated from numerical data obtained by integration of model equations (2). The continuous line represents Eq. (3) for (a) $\epsilon=0.060 \pm 3 \%$, (b) $\epsilon=0.027 \pm 4 \%$, and (c) $\epsilon=0.019 \pm 3.5 \%$, corresponding, respectively, to the intermittent states preceded by period-3, period-2, and period- 4 states shown in Figs. 2(d), 4(d), and 5(c). 
in $\mu$ s the new values of the bifurcation parameter are, respectively, $\quad \epsilon^{\prime}=0.326 \pm 3 \% ; \quad \epsilon^{\prime}=0.184 \pm 4 \% ; \quad$ and $\epsilon^{\prime}=0.051 \pm 3.5 \%$. The first two of these values are of the same order of magnitude as the corresponding ones obtained in [5] by fitting Eq. (3) to points calculated from experimental data. The distribution of the laminar-phase durations for the intermittent state preceded by a period4 state was not measured in Ref. [5]. It is probably difficult to get a better quantitative agreement between theory and experiment, taking into account that some experimental parameters (e.g., the resonator detuning) are not exactly known, and also that the real laser is a much more complex system than the one considered in our model.

\section{CONCLUSIONS AND OUTLOOK}

We have presented a theoretical study of the dynamics of type-III intermittency in a coherently pumped fourlevel laser, with pump and laser fields having crossed linear polarizations. Our model shows a family of transitions to chaos by type-III intermittencies preceded by period- $N$ states $(N=2,3,4)$ of the same type as the ones observed experimentally, though time inverted in some cases. We have not found the transition involving the period-5 state which appeared also in the experiment.

Numerically these transitions originate in a period- $N$ window in the chaotic domain. This results in two distinctive features of our results with respect to the experimental findings. First, our transitions to chaos occur over intervals of the control parameters narrower than the experimental ones. Second, our period- $N$ state is not preceded generally by a period-1 state, as in the experiment, but by an inverse sequence of period-doubling bifurcations leading to chaos. A closer fitting of theory and experiment would require both a lengthier exploration of the parameter space and the inclusion in the model of the inhomogeneous Doppler broadening, which is another important physical factor for the $\mathrm{NH}_{3}$ laser.

\section{ACKNOWLEDGMENTS}

The authors wish to thank Ramón Vilaseca for interesting discussions and a critical reading of the manuscript. This work was supported by the Dirección General de Investigación Científica y Técnica (Spain), Project No. PB89-0319.
[1] P. Manneville and Y. Pomeau, Phys. Lett. 75A, 1 (1979); Y. Pomeau and P. Manneville, Physica D 1, 219 (1980); P. Bergé, Y. Pomeau, and C. Vidal, Order within Chaos (Hermann, Paris, 1984).

[2] Among the abundant bibliography on laser dynamics, see, e.g., C. O. Weiss and R. Vilaseca, Dynamics of Lasers (VCH, Weinheim, 1991); N. B. Abraham, P. Mandel, and L. M. Narducci, in Progress in Optics $X X V$, edited by E. Wolf (Elsevier, Amsterdam, 1988), pp. 3-190.

[3] C. O. Weiss and J. Brock, Phys. Rev. Lett. 57, 2804 (1986); C. O. Weiss, N. B. Abraham, and U. Hübbner, ibid. 61, 1587 (1988); U. Hübner, N. B. Abraham, and C. O. Weiss, Phys. Rev. A 40, 6354 (1989); M. Y. Lí et al., Opt. Commun. 80, 119 (1990).

[4] C. T. Sparrow, The Lorenz Equations: Bifurcations, Chaos and Strange Attractors (Springer-Verlag, Berlin, 1982); H. Haken, Phys. Lett. 53A, 77 (1975).

[5] D. Y. Tang, J. Pujol, and C. O. Weiss, Phys. Rev. A 44, R35 (1991).
[6] D. Y. Tang, M. Y. Lí, and C. O. Weiss, Phys. Rev. A 46, 676 (1992).

[7] J. Sacher, W. Elsäser, and E. O. Göbel, Phys. Rev. Lett. 63, 2224 (1989).

[8] J. Pujol, F. Laguarta, R. Vilaseca, and R. Corbalán, J. Opt. Soc. Am. B 5, 1004 (1988); W. Forysiak, J. V. Moloney, and R. G. Harrison, Physica D 53, 162 (1991), and references therein.

[9] E. H. M. Hogenboom, W. Klische, C. O. Weiss, and A. Godone, Phys. Rev. Lett. 55, 2571 (1985); M. P. Sassi, M. Barbeau, and C. O. Weiss, Appl. Phys. B 43, 179 (1987).

[10] M. Arjona, R. Corbalán, F. Laguarta, J. Pujol, and R. Vilaseca, Phys. Rev. A 41, 6559 (1990).

[11] R. Corbalán, R. Vilaseca, M. Arjona, J. Pujol, E. Roldan, and G. J. de Valcárcel, Phys. Rev. A 48, 1483 (1993).

[12] M. Sargent III, M. O. Scully, and W. E. Lamb, Jr., Laser Physics (Addison-Wesley, Reading, MA, 1974).

[13] H. G. Schuster, Deterministic Chaos (VCH, Weinheim, 1989). 